Original Research Paper

\title{
Double Weighted Integrals Identities of Montgomery for Differentiable Function of Higher Order
}

\author{
${ }^{1}$ Asif R. Khan and ${ }^{1,2}$ Faraz Mehmood \\ ${ }^{1}$ Mathematics, University of Karachi, Pakistan \\ ${ }^{2}$ Mathematics, Dawood University of Engineering and Technology, Pakistan
}

Article history

Received: 11-11-2018

Revised: 18-01-2019

Accepted: 24-06-2019

Corresponding Author:

Faraz Mehmood

Mathematics, Dawood

University of Engineering and

Technology, Pakistan

Email: farazmehmood@yahoo.com

\section{Introduction and Preliminaries}

The topic of Montgomery's identities have many applications and cover other important known identities and inequalities in that involve Ostrowski and Gru"ss type inequalities, also see in present paper. There are many applications of Ostrowski inequalities in field of numerical integrations and probability theory (Dragomir et al., 2002; 2008; Liu, 2012). We can also get especial means using such inequalities (Alomari and Darus, 2010a; 2010b). The especial case of Ostrowski type inequalities is Čebyšev inequality which is very popular (Pečarić, 1984; 1987). Also there are many applications of Grüss type inequalities in the numerical integrations and other different fields (Buse et al., 2006; Cerone and Dragomir, 2007; Dragomir, 2012).

$J$ is an interval in $\mathbb{R}$, throughout the article. Also throughout the article we would use the following notations for PDF $=$ probability density function, $\mathbb{R}=$ $(-\infty, \infty)$ and $\mathbb{R}_{*}=[0, \infty)$. The following identity of Montgomery is extracted from (Mitrinović et al., 1991; Pečarić and Vukelić, 2007).

\section{Proposition 1.1.}

Let $f$ be a function and provided that $f$ ' is continuous in the interval $[a, b] \subset \mathbb{R}$. Then:

$$
(b-a) f(y)=\int_{a}^{b} f(p) d p+\int_{a}^{b} r(y, p) f^{\prime}(p) d p,
$$

holds for Peano kernel $r$, stated as:

$$
r(y, p)= \begin{cases}p-a, & a \leq p \leq y \\ p-b, & y<p \leq b .\end{cases}
$$

We also present here the generalization of above Equation (1.1) which is collected from (Pečarić, 1980).

\section{Proposition 1.2}

Let $f$ be a function and provided that $f^{\prime}$ is continuous in the interval $[a, b]$. Then:

$$
f(y)=\int_{a}^{b} \mathrm{v}(p) f(p) d p+\int_{a}^{b} r_{\mathrm{v}}(y, p) f^{\prime}(p) d p,
$$

holds for weighted Peano kernel $r_{v^{\prime}}$ defined as:

$$
r_{v}(y, p)= \begin{cases}V(p) & , \quad a \leq p \leq y, \\ V(p)-1, & y<p \leq b,\end{cases}
$$

where, $v:[a, b] \rightarrow \mathbb{R}_{*}$ is some PDF, i.e., it is $a$ function that satisfies $\int_{a}^{b} v(p) d p=1$ and:

$$
V(p)=\left\{\begin{array}{ccc}
0 & , & p<a \\
\int_{a}^{p} v(v) d v & , & p \in[a, b] \\
1 & , & p>b .
\end{array}\right.
$$

The following generalized identities are obtained from (Barnett and Dragomir, 2001) and (Dragomir et al., 2003) for functions of two independent variables. 


\section{Proposition 1.3}

Let $f$ be a function and provided that $f_{(1,1)}$ is continuous in the interval $[a, b] \times[c, d]$. Then:

$$
\begin{aligned}
& (d-c)(b-a) f(y, z)=-\int_{a}^{b} \int_{c}^{d} f(p, s) d s d p+(d-c) \\
& \int_{a}^{b} f(p, z) d p \\
& +(b-a) \int_{c}^{d} f(y, s) d s+\int_{a}^{b} \int_{c}^{d} r(y, p) q(z, s) f_{(1,1)}(p, s) d s d p, \\
\text { and } & (d-c)(b-a) f(y, z)=\int_{a}^{b} \int_{c}^{d} f(p, s) d s d p \\
+ & \int_{a}^{b} \int_{c}^{d} r(y, p) f_{(1,0)}(p, s) d s d p \\
+ & \int_{a}^{b} \int_{c}^{d} q(z, s) f_{(0,1)}(p, s) d s d p+\int_{a}^{b} \int_{c}^{d} r(y, p) q(z, s) \\
& f_{(1,1)}(p, s) d s d p,
\end{aligned}
$$

hold, here $r$ and $q$ are Peano kernals as defined above.

Pečarić and Vukelić (2007) authors gave the identities of weighted Montgomery for two variables functions.

\section{Proposition 1.4}

Let $r:[a, b] \times[c, d] \rightarrow \mathbb{R}$ be integrable function and $Q$ is stated as:

$$
Q(y, z)=\int_{y}^{b} \int_{z}^{d} r(v, \varsigma) d \varsigma d v
$$

If $f$ be a function and provided that $f_{(1,1)}$ is continuous in the interval $[a, b] \times[c, d]$. Then:

$$
\begin{aligned}
& Q(a, c) f(y, z)=\int_{a}^{b} \int_{c}^{d} r(p, s) f(p, s) d s d p \\
& +\int_{a}^{b} \hat{Q}(y, p) f_{(1,0)}(p, z) d p \\
& +\int_{c}^{d} \tilde{Q}(z, s) f_{(0,1)}(y, s) d s \\
& -\int_{a}^{b} \int_{c}^{l} \bar{Q}(y, p, z, s) f_{(1,1)}(p, s) d s d p,
\end{aligned}
$$

holds:

$$
\begin{aligned}
& \text { where } \hat{Q}(y, p)= \begin{cases}\int_{a}^{p} \int_{c}^{l} r(v, \zeta) d \zeta d v & , a \leq p \leq y, \\
-Q(p, c) & , y<p \leq b,\end{cases} \\
& \tilde{Q}(z, s)= \begin{cases}\int_{a}^{b} \int_{c} r(v, \zeta) d \zeta d v & , c \leq s \leq z, \\
-Q(a, s) & , z<s \leq d,\end{cases} \\
& \text { and } \bar{Q}(y, p, z, s)=\left\{\begin{array}{lll}
\int_{a}^{p} \int_{c}^{s} r(v, \zeta) d \zeta d v, & a \leq p \leq y, & c \leq s \leq z, \\
-\int_{p}^{b} \int_{c}^{s} r(v, \zeta) d \zeta d v, & y<p \leq b, & c \leq s \leq z, \\
-\int_{a}^{p} \int_{s}^{l} r(v, \zeta) d \zeta d v, & a \leq p \leq y, & z<s \leq d, \\
Q(p, s), & y<p \leq b, & z<s \leq d,
\end{array}\right.
\end{aligned}
$$

\section{Proposition 1.5}

Let the suppositions of Proposition 1.4 be true, then identity:

$$
\begin{aligned}
& Q(a, c) f(y, z)=-\int_{a}^{b} \int_{c}^{d} r(p, s) f(p, s) d s d p \\
& +\int_{a}^{b} \int_{c}^{d} r(p, s) f(p, z) d s d p \\
& +\int_{a}^{b} \int_{c}^{d} r(p, s) f(y, s) d s d p \\
& +\int_{a}^{b} \int_{c}^{d} \bar{Q}(y, p, z, s) f_{(1,1)}(p, s) d s d p,
\end{aligned}
$$

holds, where $\bar{Q}$ is as defined in Proposition 1.4.

\section{Proposition 1.6}

Let the suppositions of Proposition 1.4 be true, then:

$$
\begin{aligned}
& {[Q(a, c)]^{2} f(y, z)=Q(a, c) \int_{a}^{b} \int_{c}^{d} r(p, s) f(p, s) d s d p} \\
& +\int_{a}^{b}\left(\int_{a}^{b} \int_{c}^{d} r(v, s) \hat{Q}(y, p) f_{(1,0)}(p, s) d s d p\right) d v \\
& +\int_{c}^{d}\left(\int_{a}^{b} \int_{c}^{d} r(p, \zeta) \tilde{Q}(z, s) f_{(0,1)}(p, s) d s d p\right) d \zeta \\
& +\int_{a}^{b} \int_{c}^{d} \breve{Q}(y, p, z, s) f_{(1,1)}(p, s) d s d p,
\end{aligned}
$$

holds, where $\bar{Q}, \tilde{Q}$ and $\bar{Q}$ are defined in Proposition 1.4 and:

$$
\breve{Q}(y, p, z, s)=2 \hat{Q}(y, p) \tilde{Q}(z, s)-Q(a, c) \bar{Q}(y, p, z, s) .
$$

The pattern of present paper is divided into four parts. The first part consists on introduction and preliminaries. In the second part, we would give generalized identities of Montgomery for higher order differentiable function of two independent variables. In the 3rd and 4th parts respectively we also get the generalization of Ostrowski and Grüss type inequalities for differentiable functions of higher order for two independent variables by applying identities which proved in 2nd part. Those identities and inequalities give the generalization of many important results (Barnett and Dragomir, 2001; Dragomir et al., 2003; 2000; Guezane-Lakoud and Aissaoui, 2011; Ostrowski, 1938; Pečarić and Vukelić, 2007).

\section{Weighted Integrals Identities of Montgomery for Differentiable Function of Higher Order for Two Independent Variables}

In starting of this part, we would like to state some notations for simplification of the lengthy expressions as:

$$
Q_{\left(\begin{array}{l}
a \\
c
\end{array}\right)=\left(\begin{array}{l}
b \\
d
\end{array}\right)}^{(b,)}(y, z)=\int_{a}^{b} \int_{c}^{l} r(v, \zeta) \frac{(y-v)^{j}}{j !} \frac{(z-\eta)^{l}}{l !} d \zeta d v
$$




$$
\begin{aligned}
& Q_{\left(\begin{array}{l}
(0, l) \\
c
\end{array}\right) \Rightarrow\left(\begin{array}{l}
b \\
d
\end{array}\right)}^{(z)}(z)=\int_{a}^{b} \int_{c}^{l} r(v, \xi) \frac{(z-\zeta)^{l}}{l !} d \zeta d v, \\
& Q_{\left(\begin{array}{c}
a \\
c
\end{array}\right)=\left(\begin{array}{c}
b \\
d
\end{array}\right)}^{(j)}(y)=\int_{a}^{b} \int_{c}^{l} r(v, \zeta) \frac{(y-v)^{j}}{j !} d \zeta d v, \\
& R(f: y, z)=-\sum_{j=1}^{M} \sum_{l=1}^{N}(-1)^{j+1} f_{(j, l)}(y, z) Q_{\left(\begin{array}{l}
a \\
c
\end{array}\right) \Rightarrow\left(\begin{array}{l}
b \\
d
\end{array}\right)}^{(j, l)}(y, z) \\
& -\sum_{l=1}^{N}(-1)^{l} f_{(0, l)}(y, z) Q_{\left(\begin{array}{l}
(0, l) \\
c
\end{array}\right) \Rightarrow\left(\begin{array}{l}
b \\
d
\end{array}\right)}^{(z)}(z) \\
& -\sum_{j=1}^{M}(-1)^{j} f_{(j, 0)}(y, z) Q_{\left(\begin{array}{l}
c \\
c
\end{array}\right) \Rightarrow\left(\begin{array}{l}
b \\
d
\end{array}\right)}^{\left(\begin{array}{c}
b \\
d
\end{array}\right)}(y) \text {. }
\end{aligned}
$$

For our next main theorem of this recent part, we use a lemma from (Adnan et al., 2017) by using new notations as follows.

\section{Lemma 2.1}

Let $f$ has continuous partial derivatives $f_{(j, l)}$ and $r_{2} f$ : $[a, b] \times[c, d] \rightarrow \mathbb{R}$ be both integrable functions, where $j \in$ $\{0,1, \ldots, M, M+1\}, l \in\{0,1, \ldots, N, N+1\}$, then:

$$
\begin{aligned}
& \int_{a}^{b} \int_{c}^{d} r(y, z) f(y, z) d z d y \\
& =\sum_{j=0}^{M} \sum_{l=0}^{N} Q_{\left(\begin{array}{c}
a \\
c \\
c
\end{array}\right) \Rightarrow\left(\begin{array}{c}
b \\
d
\end{array}\right)}^{(b, d)}(-1)^{j+1} f_{(j, l)}(b, d)
\end{aligned}
$$

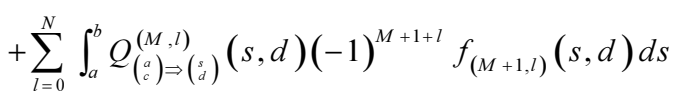

$$
\begin{aligned}
& +\sum_{j=0}^{M} \int_{c}^{d} Q_{\left(\begin{array}{c}
a \\
c
\end{array}\right) \Rightarrow\left(\begin{array}{l}
b \\
t
\end{array}\right)}^{\left(\begin{array}{l}
b \\
\text { (n) }
\end{array}\right.}(b, t)(-1)^{j+N+1} f_{(j, N+1)}(b, t) d t \\
& +\int_{a}^{b} \int_{c}^{d} Q_{\left(\begin{array}{c}
a \\
c
\end{array}\right)=\left(\begin{array}{c}
s \\
(i)
\end{array}\right)}^{(s, N)}(s, t)(-1)^{M+N} f_{(M+1, N+1)}(s, t) d t d s .
\end{aligned}
$$

Now, at this stage we are ready to give our important new theorems as follows.

\section{Theorem 2.2}

Let $f$ has continuous partial derivatives $f_{(j, l)}$ and $r, f$ : $[a, b] \times[c, d] \rightarrow \mathbb{R}$ be both integrable functions, where $j \in$ $\{0,1, \cdots, M, M+1\}, l \in\{0,1, \ldots, N, N+1\}$, then:

$$
\begin{aligned}
f(y, z) Q(b, d) & =R(f: y, z)+\int_{d}^{b} \int_{d}^{l} r(s, t) f(s, t) d t d s \\
& +\sum_{l=0}^{N} \int_{l}^{b}(-1)^{M+1+1} \hat{Q}^{(M, 1)}(y, s, z) f_{(M+1, l)}(s, z) d s \\
& +\sum_{j=0}^{M} \int_{d}^{l}(-1)^{j+s+1+1} \tilde{Q}^{(j, N)}(y, z, t) f_{(j, N+1)}(y, t) d t \\
& -\int_{d}^{l} \int_{d}^{l}(-1)^{M+1} \bar{Q}^{(M, N)}(y, s, z, t) f_{(M+1, N+1)}(s, t) d t d s,
\end{aligned}
$$

where:

$$
\begin{aligned}
& \hat{Q}^{(M, l)}(y, s, z)=\left\{\begin{array}{c}
-Q_{\left(\begin{array}{c}
M \\
c \\
c
\end{array}\right) \Rightarrow\left(\begin{array}{l}
s \\
d
\end{array}\right)}^{(M, l)}(s, z), a \leq s \leq y, \\
Q_{\left(\begin{array}{l}
s, l) \\
c
\end{array}\right) \Rightarrow\left(\begin{array}{l}
b \\
d
\end{array}\right)}^{(s, z)}, y<s \leq b,
\end{array}\right.
\end{aligned}
$$

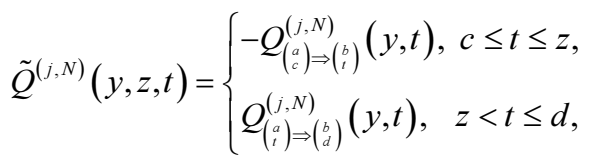

and:

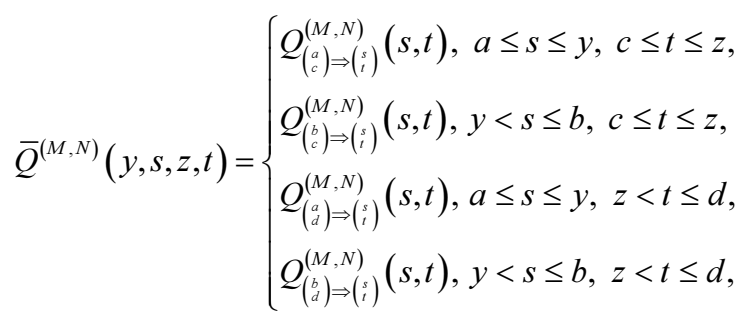

where, $Q_{(:)=(:)}^{(M, N)}(s, t), Q_{(:) \Rightarrow(:)}^{(M, l)}(s, z), Q_{(:) \Rightarrow(:)}^{(j, N)}(y, t), R(f: y, z)$ and $R(f: y, z)$ are defined in (2.1), (2.2), (2.3) and (2.4) respectively.

\section{Proof}

Using Lemma 2.1 for $[\mathrm{a}, \mathrm{y}] \times[c, z]$ we get:

$$
\begin{aligned}
& \int_{a}^{y} \int_{c}^{z} r(s, t) f(s, t) d t d s
\end{aligned}
$$

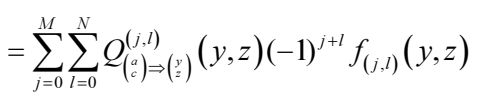

$$
\begin{aligned}
& +\sum_{l=0}^{N} \int_{a}^{y} Q_{\left(\begin{array}{l}
a \\
(
\end{array}\right) \Rightarrow\left(\begin{array}{l}
s, l) \\
z
\end{array}\right)}^{(s, z)(-1)^{M+1+l}} f_{(M+1, l)}(s, z) d s
\end{aligned}
$$

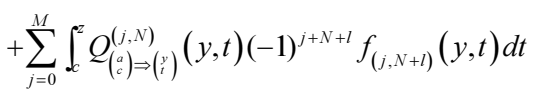

$$
\begin{aligned}
& +\int_{a}^{y} \int_{c}^{z} Q_{\left(\begin{array}{c}
a \\
c
\end{array}\right) \Rightarrow\left(\begin{array}{l}
s \\
t
\end{array}\right)}^{(M, N)}(s, t)(-1)^{N+M} f_{(M+1, N+1)}(s, t) d t d s \\
& =\sum_{j=0}^{M} \sum_{l=0}^{N}(-1)^{j+l} f_{(j, l)}(y, z)\left[Q_{\left(\begin{array}{c}
b \\
d
\end{array}\right) \Rightarrow\left(\begin{array}{l}
a \\
c
\end{array}\right)}^{(j, l)}(y, z)-Q_{\left(\begin{array}{c}
b \\
d
\end{array}\right) \Rightarrow\left(\begin{array}{l}
a \\
z
\end{array}\right)}^{(j, l)}(y, z)\right. \\
& -Q_{\left(\begin{array}{c}
b \\
d
\end{array}\right) \Rightarrow\left(\begin{array}{l}
(j, l) \\
c
\end{array}\right)}^{(y, z)}\left(y, z Q_{\left(\begin{array}{c}
b \\
d
\end{array}\right) \Rightarrow\left(\begin{array}{l}
y \\
z
\end{array}\right)}^{(j, l)}(y, z)\right]+\sum_{l=0}^{N} \int_{a}^{y}(-1)^{M+1+l} f_{(M+1, l)}(s, z) \\
& {\left[Q_{\left(\begin{array}{l}
b \\
d
\end{array}\right) \Rightarrow\left(\begin{array}{c}
a \\
c
\end{array}\right)}^{(M, l)}(s, z)-Q_{\left(\begin{array}{c}
b \\
d
\end{array}\right) \Rightarrow\left(\begin{array}{c}
a \\
z
\end{array}\right)}^{(M, l)}(s, z)-Q_{\left(\begin{array}{c}
b \\
d
\end{array}\right) \Rightarrow\left(\begin{array}{l}
s \\
c
\end{array}\right)}^{(M, l)}(s, z)+Q_{\left(\begin{array}{c}
b \\
d
\end{array}\right) \Rightarrow\left(\begin{array}{l}
s \\
z
\end{array}\right)}^{(M, l)}(s, z)\right] d s} \\
& +\sum_{j=0}^{M} \int_{c}^{z}(-1)^{j+N+1} f_{(j, N+1)}(y, t) \times\left[Q_{\left(\begin{array}{l}
b \\
d
\end{array}\right) \Rightarrow\left(\begin{array}{l}
a \\
c
\end{array}\right)}^{(j,)}(y, t)-Q_{\left(\begin{array}{l}
b \\
d
\end{array}\right) \Rightarrow\left(\begin{array}{l}
a \\
t
\end{array}\right)}^{(j, N)}(y, t)\right. \\
& \left.-Q_{\left(\begin{array}{l}
b \\
d
\end{array}\right) \Rightarrow\left(\begin{array}{l}
y \\
c
\end{array}\right)}^{(j, N)}(y, t)+Q_{\left(\begin{array}{l}
b \\
d
\end{array}\right) \Rightarrow\left(\begin{array}{l}
y \\
t
\end{array}\right)}^{(j)}(y, t)\right] d t+\int_{a}^{y} \int_{c}^{z}(-1)^{N+M} f_{(M+1, N+1)}(s, t) \\
& {\left[Q_{\left(\begin{array}{l}
b \\
d
\end{array}\right) \Rightarrow\left(\begin{array}{l}
a \\
c
\end{array}\right)}^{(M, N)}(s, t)-Q_{\left(\begin{array}{l}
b \\
d
\end{array}\right) \Rightarrow\left(\begin{array}{l}
a \\
t
\end{array}\right)}^{(M, N)}(s, t)-Q_{\left(\begin{array}{l}
b \\
d
\end{array}\right) \Rightarrow\left(\begin{array}{l}
s \\
c
\end{array}\right)}^{(M, N)}(s, t)+Q_{\left(\begin{array}{l}
b \\
d
\end{array}\right) \Rightarrow\left(\begin{array}{l}
s \\
t
\end{array}\right)}^{(M, N)}(s, t)\right] d t d s .}
\end{aligned}
$$

Similarly for $[y, b] \times[c, z]$ we get:

$$
\begin{aligned}
& \int_{y}^{b} \int_{c}^{z} r(s, t) f(s, t) d t d s=-\int_{b}^{y} \int_{c}^{z} r(s, t) f(s, t) d t d s \\
& =-\sum_{j=0}^{M} \sum_{l=0}^{N}(-1)^{j+l} f_{(j, l)}(y, z)\left[-Q_{\left(\begin{array}{c}
b \\
d
\end{array}\right) \Rightarrow\left(\begin{array}{l}
(j) \\
c
\end{array}\right)}(y, z)+Q_{\left(\begin{array}{c}
b \\
d
\end{array}\right) \Rightarrow\left(\begin{array}{l}
(j) \\
z
\end{array}\right)}(y, z)\right]
\end{aligned}
$$




$$
\begin{aligned}
& +\sum_{l=0}^{N} \int_{y}^{b}(-1)^{M+1+l} f_{(M+1, l)}(s, z)\left[-Q_{\left(\begin{array}{l}
b \\
d
\end{array}\right)=\left(\begin{array}{l}
(s) \\
c
\end{array}\right)}^{(s, l)}(s)+Q_{\left(\begin{array}{l}
b \\
d
\end{array}\right) \Rightarrow\left(\begin{array}{l}
s \\
z
\end{array}\right)}^{(M, l)}(s, z)\right] d s \\
& -\sum_{j=0}^{M} \int_{d}^{z}(-1)^{j+N+1} f_{(j, N+1)}(y, t)\left[-Q_{\left(\begin{array}{l}
b \\
d
\end{array}\right) \Rightarrow\left(\begin{array}{l}
y \\
c
\end{array}\right)}^{(j, N)}(y, t)+Q_{\left(\begin{array}{l}
b \\
d
\end{array}\right) \Rightarrow\left(\begin{array}{l}
y \\
t
\end{array}\right)}^{(j, N)}(y, t)\right] d t \\
& +\int_{y}^{b} \int_{d}^{z}(-1)^{N+M} f_{(M+1, N+1)}(s, t)\left[-Q_{\left(\begin{array}{l}
b \\
d
\end{array}\right)=\left(\begin{array}{l}
s \\
c
\end{array}\right)}^{(s, N)}(s, t)+Q_{\left(\begin{array}{l}
b \\
d
\end{array}\right)=\left(\begin{array}{l}
s \\
t
\end{array}\right)}^{(M, N)}(s, t)\right] d t d s .
\end{aligned}
$$

For $[a, y] \times[z, d]$ :

$$
\begin{aligned}
& \int_{a}^{y} \int_{z}^{d} r(s, t) f(s, t) d t d s=-\int_{a}^{y} \int_{d}^{z} r(s, t) f(s, t) d t d s
\end{aligned}
$$

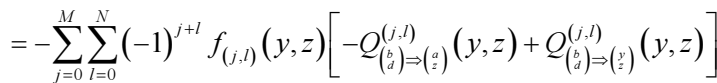

$$
\begin{aligned}
& -\sum_{l=0}^{N} \int_{a}^{y}(-1)^{M+1+l} f_{(M+1, l)}(s, z)\left[-Q_{\left(\begin{array}{c}
\left(\begin{array}{c}
b \\
d
\end{array}\right) \Rightarrow\left(\begin{array}{l}
a \\
z
\end{array}\right) \\
(M, l)
\end{array}\right.}(s, z)+Q_{\left(\begin{array}{l}
b \\
d
\end{array}\right) \Rightarrow\left(\begin{array}{l}
s \\
z
\end{array}\right)}^{(M, l)}(s, z)\right] d s \\
& +\sum_{j=0}^{M} \int_{z}^{l}(-1)^{j+N+1} f_{(j, N+1)}(y, t)\left[-Q_{\left(\begin{array}{l}
b \\
d
\end{array}\right) \Rightarrow\left(\begin{array}{l}
a \\
t
\end{array}\right)}^{(j, N)}(\mathrm{y}, t)+Q_{\left(\begin{array}{l}
b \\
d
\end{array}\right) \Rightarrow\left(\begin{array}{l}
y \\
t
\end{array}\right)}^{(j, N)}(\mathrm{y}, \mathrm{t})\right] d t \\
& +\int_{a}^{y} \int_{z}^{d}(-1)^{M+N} f_{(M+1, N+1)}(s, t)\left[-Q_{\left(\begin{array}{l}
b \\
d
\end{array}\right) \Rightarrow\left(\begin{array}{l}
a \\
t
\end{array}\right)}^{(M, N)}(s, t)+Q_{\left(\begin{array}{l}
b \\
d
\end{array}\right) \Rightarrow\left(\begin{array}{l}
s \\
t
\end{array}\right)}^{(M, N)}(s, t)\right] d t d s .
\end{aligned}
$$

At last for $[y, b] \times[z, d]$ :

$$
\begin{aligned}
& \int_{y}^{b} \int_{z}^{d} r(s, t) f(s, t) d t d s=\int_{b}^{y} \int_{d}^{z} r(s, t) f(s, t) d t d s \\
& =\sum_{j=0}^{M} \sum_{l=0}^{N}(-1)^{j+l} f_{(j, l)}(y, z) Q_{\left(\begin{array}{c}
b \\
d
\end{array}\right) \Rightarrow\left(\begin{array}{l}
y \\
z
\end{array}\right)}^{(j, l)}(y, z) \\
& -\sum_{l=0}^{N} \int_{y}^{b}(-1)^{M+1+l} f_{(M+1, l)}(s, z)+Q_{\left(\begin{array}{c}
b \\
d
\end{array}\right) \Rightarrow\left(\begin{array}{l}
s \\
z
\end{array}\right)}^{(M, l)}(s, z) d s \\
& -\sum_{j=0}^{M} \int_{z}^{d}(-1)^{j+N+1} f_{(j, N+1)}(y, t) Q_{\left(\begin{array}{c}
b \\
d
\end{array}\right) \Rightarrow\left(\begin{array}{l}
y \\
t
\end{array}\right)}^{(j, N)}(\mathrm{y}, t) d t \\
& +\int_{y}^{b} \int_{c}^{z}(-1)^{M+N} f_{(M+1, N+1)}(s, t) Q_{\left(\begin{array}{c}
b \\
d \\
d
\end{array}\right) \Rightarrow\left(\begin{array}{l}
s \\
t
\end{array}\right)}^{(s, N)}(s, t) d t d s .
\end{aligned}
$$

Adding the four expressions we get desired result.

\section{Theorem 2.3}

Let $f$ has continuous partial derivatives $f_{(M+1, N+1)}$ and $f, r$ : $[a, b] \times[c, d] \rightarrow \mathbb{R}$ be both integrable functions. Then following identity:

$$
\begin{aligned}
& f(y, z) Q(b, d)=R(f: y, z)-\int_{a}^{b} \int_{c}^{d} r(s, t) f(s, t) d t d s \\
& +\int_{a}^{b} \int_{c}^{d} r(s, t) f(s, z) d t d s+\int_{a}^{b} \int_{c}^{l} r(s, t) f(y, t) d t d s \\
& +\sum_{l=1}^{N} \int_{a}^{b} \int_{c}^{d}(-1)^{l} r(s, \zeta) \frac{(\zeta-z)^{l}}{l !} f_{(0, l)}(s, z) d \varsigma d s \\
& +\sum_{j=1}^{M} \int_{a}^{b} \int_{c}^{d}(-1)^{j} r(v, t) \frac{(v-y)^{j}}{j !} f_{(j, 0)}(y, t) d t d v \\
& +\int_{a}^{b} \int_{c}^{d}(-1)^{N+M} \bar{Q}^{(M, N)}(y, s, z, t) f_{(M+1, N+1)}(s, t) d t d s,
\end{aligned}
$$

holds, where $\bar{Q}^{(M, N)}(y, s, z, t)$ as in Theorem 2.2 and $Q(b, d)$ and $R(f: y, z)$ are as defined in (1.2) and (2.3) respectively.

\section{Proof}

Firstly, finding expression for the following:

$$
\int_{a}^{b}(-1)^{M+1+l} \hat{Q}^{(M, l)}(y, s, z) f_{(M+1, l)}(s, z) d s
$$

using integration by parts as:

$$
\begin{aligned}
& \int_{c}^{l}(-1)^{M+1+l} \hat{Q}^{(M, l)}(y, s, z) f_{(M+1, l)}(s, z) d s \\
& =-\int_{a}^{y}(-1)^{M+1+l} Q_{\left(\begin{array}{l}
a \\
c
\end{array}\right) \Rightarrow\left(\begin{array}{l}
s \\
d
\end{array}\right)}^{(M, l)}(s, z) f_{(M+1, l)}(s, z) d s \\
& +\int_{y}^{b}(-1)^{M+1+l} Q_{\left(\begin{array}{c}
s \\
c
\end{array}\right) \Rightarrow\left(\begin{array}{l}
b \\
d
\end{array}\right)}^{(M, l)}(s, z) f_{(M+1, l)}(s, z) d s \\
& =-\left(\int_{a}^{y}(-1)^{M+1+l} Q_{\left(\begin{array}{c}
a \\
c
\end{array}\right) \Rightarrow\left(\begin{array}{l}
s \\
d
\end{array}\right)}^{(M, l)}(s, z) f_{(M+1, l)}(s, z) d s\right. \\
& \left.+\int_{y}^{b}(-1)^{M+1+l} Q_{\left(\begin{array}{c}
b \\
c
\end{array}\right) \Rightarrow\left(\begin{array}{c}
s \\
d
\end{array}\right)}^{(M, l)}(s, z) f_{(M+1, l)}(s, z) d s\right) \\
& =Q_{\left(\begin{array}{c}
a \\
c
\end{array}\right) \Rightarrow\left(\begin{array}{l}
y \\
d
\end{array}\right)}^{(M, l)}(y, z)(-1)^{M+l} f_{(M, l)}(y, z) \\
& +\int_{a}^{y} Q_{\left(\begin{array}{c}
a \\
c
\end{array}\right) \Rightarrow\left(\begin{array}{c}
s \\
d
\end{array}\right)}^{(M-1, l)}(s, z)(-1)^{M+l} f_{(M, l)}(s, z) d s \\
& +Q_{\left(\begin{array}{c}
y \\
c
\end{array}\right) \Rightarrow\left(\begin{array}{l}
b \\
d
\end{array}\right)}^{\left(\begin{array}{l}
b \\
\text { (n) }
\end{array}\right)}(y, z)(-1)^{M+l} f_{(M, l)}(y, z)
\end{aligned}
$$

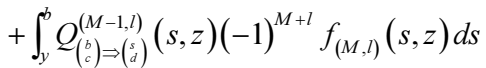

$$
\begin{aligned}
& =Q_{\left(\begin{array}{l}
a \\
c \\
c
\end{array}\right) \Rightarrow\left(\begin{array}{l}
b \\
d
\end{array}\right)}^{(M, l)}(y, z)(-1)^{M+l} f_{(M, l)}(y, z) \\
& +\int_{a}^{y} Q_{\left(\begin{array}{c}
a \\
c
\end{array}\right) \Rightarrow\left(\begin{array}{l}
s \\
d
\end{array}\right)}^{(M-1, l)}(s, z)(-1)^{M+l} f_{(M, l)}(s, z) d s
\end{aligned}
$$

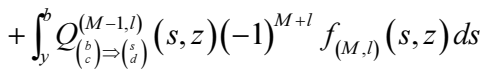

$$
\begin{aligned}
& =Q_{\left(\begin{array}{c}
a \\
c
\end{array}\right) \Rightarrow\left(\begin{array}{c}
b \\
d
\end{array}\right)}^{\left(\begin{array}{c}
b \\
d
\end{array}\right.}(y, z)(-1)^{M+l} f_{(M, l)}(y, z) \\
& +\int_{a}^{b}(-1)^{M+1} \hat{Q}^{(M-1, l)}(y, s, z) f_{(M, l)}(s, z) d s,
\end{aligned}
$$

continuing in this way we finally get:

$$
\begin{aligned}
& \int_{a}^{b}(-1)^{M+1+l} \hat{Q}^{(M, l)}(y, s, z) f_{(M+1, l)}(s, z) d s \\
& =\int_{a}^{b} \int_{c}^{l} r(v, \zeta) \frac{(\zeta-z)^{l}}{l !}\left[\sum_{i=0}^{M} \frac{(v-y)^{i}}{i !}(-1)^{i+1} f_{(i, l)}(y, z)\right] d \zeta d v \\
& -\int_{a}^{b} \int_{c}^{l} r(s, \zeta) \frac{(\zeta-z)^{l}}{l !}(-1)^{l} f_{(0, l)}(s, z) d \zeta d s .
\end{aligned}
$$

Similarly:

$$
\begin{aligned}
& \int_{c}^{l}(-1)^{j+N+1} \tilde{Q}^{(j, N)}(y, z, t) f_{(j+N+1)}(y, t) d t \\
& =\int_{a}^{b} \int_{c}^{l} r(v, \zeta) \frac{(v-y)^{j}}{j !}\left[\sum_{k=0}^{N} \frac{(\zeta-z)^{k}}{k !}(-1)^{j+k} f_{(j, k)}(y, z)\right] d \zeta d v \\
& -\int_{a}^{b} \int_{c}^{l} r(v, t) \frac{(v-y)^{j}}{j !}(-1)^{j} f_{(j, 0)}(y, t) d t d v .
\end{aligned}
$$

We get desired identity by putting all above values in (2.6) then some cancelation and rearrangements.

\section{Theorem 2.4}

Let $f:[a, b] \times[c, d] \rightarrow \mathbb{R}$ be function and $f \in C^{(2 N+1,2 M+1)}$ and $\forall(y, z) \in[a, b] \times[c, d]$ we have: 


$$
\begin{aligned}
& f(y, z) Q(b, d)^{2}=Q(b, d) R(f: y, z)+Q(b, d) \\
& \int_{a}^{b} \int_{c}^{l} r(s, t) f(s, t) d t d s+\sum_{l=0}^{N} \int_{a}^{b}(-1)^{M+1+l} \hat{Q}^{(M, l)}(y, s, z) \\
& R\left(f_{(M+1, l)}: s, z\right) d s+\sum_{j=0}^{M} \int_{c}^{t}(-1)^{j+N+1} \tilde{Q}^{(j, N)}(y, z, t) \\
& R\left(f_{(j+N+1)}: y, t\right) d t \\
& +\sum_{j=0}^{M} \sum_{l=0}^{N} \int_{a}^{b} \int_{a}^{b} \int_{c}^{d}(-1)^{M+1+j+l} \hat{Q}^{(M, l)}(y, s, z) \\
& r(v, t) \frac{(v-y)^{j}}{j !} f_{(M+1+j, l)}(s, t) d t d s d v \\
& +\sum_{j=0}^{M} \sum_{l=0}^{N} \int_{c}^{l} \int_{a}^{b} \int_{c}^{d}(-1)^{j+l+N+1} \tilde{Q}^{(j, N)}(y, z, t) \\
& r(s, \zeta) \frac{(\zeta-z)^{l}}{l !} f_{(j, N+1+l)}(s, t) d t d s d \zeta \\
& +2 \int_{a}^{b} \int_{c}^{l} \sum_{j=0}^{M} \sum_{l=0}^{N}(-1)^{j+l+M+N} \hat{Q}^{(M, l)}(y, s, z) \\
& \tilde{Q}^{(j, N)}(y, z, t) f_{(M+1+j, N+1+l)}(s, t) d t d s, \\
& -Q(b, d) \int_{a}^{b} \int_{c}^{l}(-1)^{M+N} \bar{Q}^{(M, N)}(y, s, z, t) f_{(M+1, N+1)}(s, t) d t d s,
\end{aligned}
$$

where, $\hat{Q}^{(M, l)}(y, s, z) \tilde{Q}^{(N, j)}(y, z, t) \bar{Q}^{(M, N)}(y, s, z, t), Q$ and $r$ are as in Theorem 2.2.

\section{Proof}

By Sums (2.8) for $l=0, \ldots, N$ and (2.9) for $j=0, \ldots, M$ we obtain respectively:

$$
\begin{aligned}
& f(y, z) Q(b, d)=R(f: y, z) \\
& +\sum_{l=0}^{N} \int_{a}^{b} \int_{c}^{l}(-1)^{l} r(s, \zeta) \frac{(\zeta-z)^{l}}{l !} f_{(0, l)}(s, z) d \zeta d s \\
& +\sum_{l=0}^{M} \int_{a}^{d}(-1)^{M+1+l} \hat{Q}^{(M, l)}(y, s, z) f_{(M+1, l)}(s, z) d s,
\end{aligned}
$$

and:

$$
\begin{aligned}
& f(y, z) Q(b, d)=R(f: y, z) \\
& +\sum_{j=0}^{M} \int_{a}^{b} \int_{c}^{d}(-1)^{j} r(v, t) \frac{(v-y)^{j}}{j !} f_{(j, 0)}(y, t) d t d v \\
& +\sum_{j=0}^{M} \int_{c}^{d}(-1)^{j+N+1} \tilde{Q}^{(j, N)}(y, z, t) f_{(j, N+1)}(y, t) d t,
\end{aligned}
$$

$$
\forall(y, z) \in[a, b] \times[c, d] .
$$

Formula (2.11) used for partial derivatives $f_{(, N+1)}$ for $j$ $=0,1, \ldots, M$, then:

$$
\begin{aligned}
& f_{(j, N+1)}(y, t) Q(b, d)=R\left(f_{(j, N+1)}: y, t\right) \\
& +\sum_{l=0}^{N} \int_{a}^{b} \int_{c}^{d}(-1)^{l} r(s, \zeta) \frac{(\zeta-t)^{l}}{l !} f_{(j, N+1+l)}(s, t) d \zeta d s \\
& +\sum_{l=0}^{N} \int_{a}^{b}(-1)^{M+1+l} \hat{Q}^{(M, l)}(y, s, t) f_{(M+1+j, N+1+l)}(s, t) d s .
\end{aligned}
$$

Formula (2.12) used for partial derivatives $f_{(M+1, l)}$ for $l$ $=0,1, \ldots, N$, then:

$$
\begin{aligned}
& f_{(M+1, l)}(s, z) Q(b, d)=R\left(f_{(M+1, l)}: s, z\right) \\
& +\sum_{j=0}^{M} \int_{a}^{b} \int_{c}^{d}(-1)^{j} r(v, t) \frac{(v-s)^{j}}{j !} f_{(M+1+j, l)}(s, t) d t d v \\
& +\sum_{j=0}^{M} \int_{c}^{l}(-1)^{j+N+1} \tilde{Q}^{(j, N)}(s, z, t) f_{(M+1+j, N+1+l)}(s, t) d t .
\end{aligned}
$$

Substituting (2.13) and (2.14) into (2.6) we get:

$$
\begin{aligned}
& f(y, z) Q(b, d)=R(f: y, z)+\int_{a}^{b} \int_{c}^{l} r(s, t) f(s, t) d t d s \\
& +\frac{1}{Q(b, d)} \sum_{l=0}^{N} \int_{a}^{b}(-1)^{M+1+l} \hat{Q}^{(M, l)}(y, s, z)\left[R\left(f_{(M+1, l)}: s, z\right)\right. \\
& +\sum_{j=0}^{M} \int_{a}^{b} \int_{c}^{l}(-1)^{j} r(v, t) \frac{(v-s)^{j}}{j !} f_{(M+1+j, l)}(s, t) d t d v \\
& \left.+\sum_{j=0}^{M} \int_{l}^{l}(-1)^{j+N+1} \tilde{Q}^{(j, N)}(s, z, t) f_{(M+1+j, N+1+l)}(s, t) d t\right] d s \\
& +\frac{1}{Q(b, d)} \sum_{j=0}^{M} \int_{c}^{l}(-1)^{j+N+1} \tilde{Q}^{(j, N)}(y, z, t)\left[R\left(f_{(j, N+1)}: y, t\right)\right. \\
& +\sum_{l=0}^{N} \int_{a}^{b} \int_{c}^{l}(-1)^{l} r(s, \zeta) \frac{(\zeta-t)^{l}}{l !} f_{(j, N+1+l)}(s, t) d \zeta d s \\
& \left.+\sum_{l=0}^{N} \int_{a}^{b}(-1)^{M+1+l} \hat{Q}^{(M, l)}(y, s, t) f_{(M+1+j, N+1+l)}(s, t) d s\right] d t \\
& -\int_{a}^{b} \int_{c}^{d}(-1)^{M+N} \bar{Q}^{(M, N)}(y, s, z, t) f_{(M+1, N+1)}(s, t) d t d s .
\end{aligned}
$$

We get desired result, after some rearrangements and using Theorem of Fubini.

\section{Remark 2.5}

For $M=N=0$, we can get especial cases of Theorem 2.2, 2.3 and 2.4 as similar as Proposition 1.4, 1.5 and 1.6 respectively (also see similar case in (Pečarić and Vukelić, 2007).

\subsection{Especial Cases}

If $r(s, t)=q(s) p(t)$ in identities (2.6), (2.7) and (2.10) then we obtain the following especial cases respectively:

$$
\begin{aligned}
& f(y, z) Q_{a \rightarrow b}(q) Q_{c \rightarrow d}(p)=S(f: y, z) \\
& +\int_{a}^{b} \int_{c}^{l} p(t) q(s) f(s, t) d t d s \\
& +\sum_{l=0}^{N} \int_{a}^{b}(-1)^{M+1+l} \hat{S}^{(M, l)}(y, s, z) f_{(M+1, l)}(s, z) d s \\
& +\sum_{j=0}^{M} \int_{c}^{l}(-1)^{j+N+1} \tilde{S}^{(j, N)}(y, z, t) f_{(j, N+1)}(y, t) d t \\
& -\int_{a}^{b} \int_{c}^{d} \bar{S}^{(M, N)}(y, s, z, t)(-1)^{M+N} f_{(M+1, N+1)}(s, t) d t d s,
\end{aligned}
$$




$$
\begin{aligned}
& f(y, z) Q_{a \rightarrow b}(q) Q_{c \rightarrow d}(p)=S(f: y, z) \\
& -\int_{a}^{b} \int_{c}^{d} q(s) p(t) f(s, t) d t d s \\
& +\int_{a}^{b} \int_{c}^{d} p(t) q(s) f(s, z) d t d s \\
& +\int_{a}^{b} \int_{c}^{l} p(t) q(s) f(y, t) d t d s \\
& +\sum_{l=1}^{N} \int_{a}^{b} q(s)(-1)^{l} f_{(0, l)}(s, z) d s S_{c \rightarrow d}^{(l)}(p, z) \\
& +\sum_{j=1}^{M} S_{c \rightarrow b}^{(j)}(q, y) \int_{c}^{d} p(t)(-1)^{j} f_{(j, 0)}(y, t) d t \\
& +\int_{a}^{b} \int_{c}^{t}(-1)^{M+N} \bar{S}^{(M, N)}(y, s, z, t) f_{(M+1, N+1)}(s, t) d t d s, \\
& f(y, z)\left[Q_{a \rightarrow b}(q) Q_{c \rightarrow d}(p)\right]^{2}=Q_{a \rightarrow b}(q) Q_{c \rightarrow d}(p) S(f: y, z) \\
& +Q_{a \rightarrow b}(q) Q_{c \rightarrow d}(p) \int_{a}^{b} \int_{d}^{l} q(s) p(t) f(s, t) d t d s \\
& +\sum_{l=0}^{N} \int_{l}^{b}(-1)^{M+1+l} \hat{S}^{(M, l)}(y, s, z) S\left(f_{(M+1, l)}: s, z\right) d s \\
& +\sum_{j=0}^{M} \int^{l}(-1)^{j+N+1} \tilde{S}^{(j, N)}(y, z, t) S\left(f_{(j, M+1)}: y, t\right) d t \\
& +\sum_{j=0}^{M} \sum_{l=0}^{N} S_{a \rightarrow b}^{(j)}(q, y) \int_{a}^{b} \int_{l}^{l}(-1)^{M+1+j+l} \hat{S}^{(M, l)}(y, s, z) p(t) f_{(M+1+j, l)}(s, t) d t d s \\
& +\sum_{j=0}^{M} \sum_{l=0}^{N} S_{c \rightarrow d}^{(l)}(p, z) \int_{a}^{b} \int_{c}^{l}(-1)^{j+N+1+l} \tilde{S}^{(j, N)}(y, z, t) q(s) f_{(j, N+1+l)}(s, t) d t d s \\
& +2 \int_{a}^{b} \int^{T} \sum_{j=0}^{M} \sum_{l=0}^{N}(-1)^{j+M+1++N} \hat{S}^{(M, l)}(y, s, z) \tilde{S}^{(j, N)}(y, z, t) f_{(M+1+j, N+1+1)}(s, t) d t d s \\
& -Q_{a \rightarrow b}(q) Q_{c \rightarrow d}(p) \int_{d}^{b} \int_{d}^{d}(-1)^{N+M} \bar{S}^{(M, N)}(y, s, z, t) f_{(M+1, N+1)}(s, t) d t d s,
\end{aligned}
$$

where:

$$
\begin{aligned}
& Q_{a \rightarrow b}(q)=\int_{a}^{b} q(s) d s, \quad S_{a \rightarrow b}^{(j)}(q, y)=\int_{a}^{b} q(v) \frac{(y-v)^{j}}{j !} d v, \\
& S_{(a)=\left(\begin{array}{l}
(j) \\
(j)
\end{array}\right)}^{(j, l)}(y, z)=S_{a \rightarrow b}^{(j)}(q, y) S_{c \rightarrow d}^{(l)}(p, z), \\
& S_{\left(\begin{array}{l}
a \\
c
\end{array}\right) \Rightarrow\left(\begin{array}{l}
b \\
d
\end{array}\right)}^{(0, l)}(z)=Q_{a \rightarrow b}(q) S_{c \rightarrow d}^{(l)}(p, z),
\end{aligned}
$$

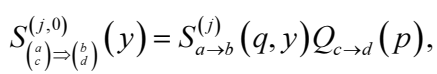

$$
\begin{aligned}
& S(f: y, z)=-\sum_{j=1}^{M} \sum_{l=1}^{N} f_{(j, l)}(y, z) S_{\left(\begin{array}{l}
a \\
c
\end{array}\right) \Rightarrow\left(\begin{array}{l}
(j) \\
(d)
\end{array}\right)}^{(y, z)}
\end{aligned}
$$

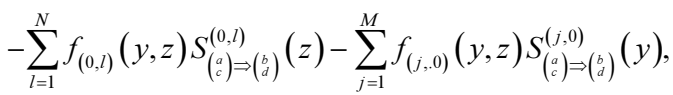

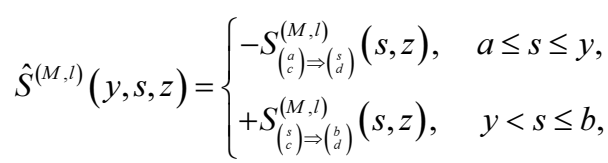

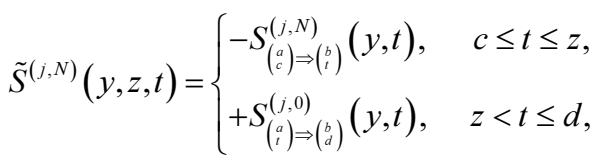

and:

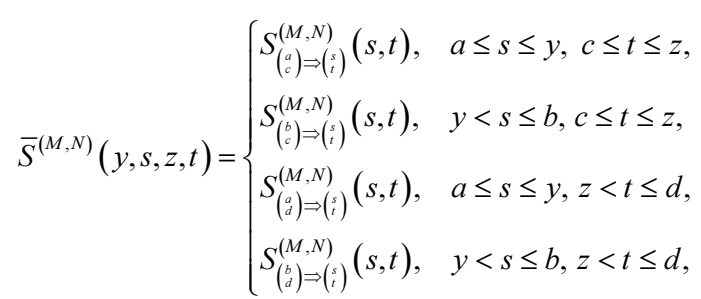

\section{Ostrowski Type Inequalities for Double Weighted Integrals for Higher Order Differentiable Function of Two Variables}

Under this heading we would recall well known Ostrowski inequality which is extracted from (Ostrowski, 1938):

$$
\begin{aligned}
& \left|f(y)-\frac{1}{b-a} \int_{a}^{b} f(t) d t\right| \\
& \leq M\left[\frac{\left(y-\frac{a+b}{2}\right)^{2}}{(b-a)^{2}}+\frac{1}{1}\right](b-a) ; y \in[b, a]
\end{aligned}
$$

where, $f$ is a real valued function in the interval $[\mathrm{a}, \mathrm{b}]$ and provided that $f^{\prime}$ is continuous and satisfying $f^{\prime}(y) \mid \leq M$, $\forall y \in[a, b]$. This inequality has helped to provide many generalizations and in Pečarić and Vukelić (2007) have also provided the generalizations of this inequality using identities (1.3) and (1.4). Now by using identities (2.6) and (2.7), we would get results for generalization of Ostrowski type for differentiable function of higher order for two variables as below.

\section{Theorem 3.1}

Let $f$ be a real valued function in the interval $[a, b] \times[c, d]$ and $f \in C^{(M+1, N+1)}$ in the same interval. Then:

$$
\begin{aligned}
& \left|f(y, z)-\frac{1}{Q(b, d)} \int_{a}^{b} \int_{c}^{d} r(s, t) f(s, t) d t d s\right| \leq O(y, z) \\
& +\sum_{l=0}^{N} \hat{O}^{(0, l)}(y, z)+\sum_{j=0}^{M} \tilde{O}^{(j, 0)}(y, z)+\bar{O}(y, z)
\end{aligned}
$$

holds, $\forall(\mathrm{y}, \mathrm{z}) \in[\mathrm{a}, \mathrm{b}] \times[\mathrm{c}, \mathrm{d}]$ where:

$$
\begin{aligned}
& O(y, z)=\frac{1}{|Q(b, d)|}|R(f: y, z)|, \\
& \hat{O}^{(0, l)}(y, z)=\frac{1}{Q(b, d) \mid}\left(\sum_{l=0}^{N} \int_{a}^{b}\left|\hat{Q}^{(M, l)}(y, s, z)\right|^{\mid \hat{l} l} d s\right)^{1 / \hat{q} l} \cdot\left\|(-1)^{M+1+l t} f_{(M+1+1)}\right\|_{j, j}, \\
& p \text { r ovided that } f_{(M+1, l)} \in L_{f_{1}(}([a, b] \times[c, d]), 1 / \tilde{r}_{l}+1 / \tilde{q}_{l}=1, \\
& \tilde{O}^{(j, 0)}(y, z)=\frac{1}{|Q(b, d)|}\left(\sum_{j=0}^{M} \int_{c}^{d}\left|\tilde{Q}^{(j, N)}(y, z, t)\right|^{\sigma_{j}} d t\right)^{1 / \tilde{\partial} j} \cdot\left\|(-1)^{j+N+1} f_{(j+N+1)}\right\| \tilde{r}_{j}, \\
& p \text { r ovided that } f_{(j, N+1)} \in L_{\tilde{r}_{j}}([a, b] \times[c, d]), 1 / \tilde{r}_{j}+1 / \tilde{q}_{j}=1, \\
& \bar{O}(y, z)=\frac{1}{|Q(b, d)|}\left(\int_{a}^{b} \int_{c}^{d}\left|\bar{Q}^{(M, N)}(y, s, z, t)\right|^{\bar{T}} d t d s\right)^{1 / \bar{T}} \cdot\left\|(-1)^{M+N} f_{(M+1, N+1)}\right\|_{T,} \\
& \text { provided that } f_{(M+1, N+1)} \in L_{\bar{F}}([a, b] \times[c, d]), 1 / \bar{r}+1 / \bar{q}=1,
\end{aligned}
$$


where, $\quad \hat{Q}^{(M, l)}(y, s, z), \tilde{Q}^{(j, N)}(y, z, t), \bar{Q}^{(M, N)}(y, s, z, t) \quad$ are $\quad$ as $\quad$ in Theorem 2.2 where as $Q$ and $R(f: y, z)$ are stated in (1.2) and (2.3) respectively.

\section{Proof}

Identity (2.6) may be written as:

$$
\begin{aligned}
& f(y, z)-\frac{1}{Q(b, d)} \int_{a}^{b} \int_{c}^{l} r(s, t) f(s, t) d t d s \\
& =\frac{1}{Q(b, d)}\left[R(f: y, z)+\sum_{l=0}^{N} \int_{a}^{b}(-1)^{M+1+l} \hat{Q}^{(M, l)}(y, s, z) f_{(M+1, l)}(s, z) d s\right. \\
& +\sum_{j=0}^{M} \int_{c}^{d}(-1)^{j+N+1} \tilde{Q}^{(j, N)}(y, z, t) f_{(j, N+1)}(y, t) d t \\
& \left.-\int_{a}^{b} \int_{c}^{d}(-1)^{M+N} \bar{Q}^{(M, N)}(y, s, z, t) f_{(M+1, N+1)}(s, t) d t d s\right] .
\end{aligned}
$$

We can easily get desired result by taking absolute value and by using inequality of Hölder for double integrals.

\section{Remark 3.2}

For $M=N=0$, we can get especial case of Theorem 3.1 as similar as Theorem 4 of (Pečarić and Vukelić, 2007).

\section{Theorem 3.3}

Let $f$ be a real valued continuous function in the interval $[a, b] \times[c, d] \exists, f \in C^{(M+1, N+1)}$ in the same interval and $\left|f_{(M+1, N+1)}\right|^{r}$ be integrable function i.e.,:

$$
\left\|(-1)^{N+M} f_{(M+1, N+1)}\right\|_{r}:=\left(\int_{a}^{b} \int_{d}^{l}\left|(-1)^{N+M} f_{(M+1, N+1)}(s, t)\right|^{r} d t d s\right)^{1 / r}<\infty,
$$

where, $1 / r+1 / q=1$. Then, it follows:

$$
\begin{aligned}
& \mid \int_{a}^{b} \int_{c}^{d} r(s, t) f(s, t) d t d s-\left[R(f: y, z)+\int_{a}^{b} \int_{c}^{d} r(s, t) f(s, z) d t d s\right. \\
& +\int_{a}^{b} \int_{c}^{d} r(s, t) f(y, t) d t d s \\
& +\sum_{l=1}^{N} \int_{a}^{b} \int_{c}^{d}(-1)^{l} r(s, \zeta) \frac{(\zeta-z)^{l}}{l !} f_{(0, l)}(s, z) d \zeta d s \\
& \left.+\sum_{j=1}^{M} \int_{a}^{b} \int_{c}^{d}(-1)^{j} r(v, t) \frac{(v-y)^{j}}{j !} f_{(j, 0)}(y, t) d t d v-f(y, z) Q(b, d)\right] \\
& \leq\left(\int_{a}^{b} \int_{c}^{d}\left|\bar{Q}^{(M, N)}(y, s, z, t)\right|^{r} d t d s\right)^{1 / r}\left\|(-1)^{M+N} f_{(M+1, N+1)}\right\|_{q} .
\end{aligned}
$$

for all $(y, z) \in[a, b] \times[c, d]$.

\section{Proof}

Identity (2.7) can be written like:

$$
\begin{aligned}
& \int_{a}^{b} \int_{c}^{d} f(s, t) r(s, t) d t d s-\left[R(f: y, z)+\int_{a}^{b} \int_{c}^{d} r(s, t) f(s, z) d t d s\right. \\
& +\int_{a}^{b} \int_{c}^{d} r(s, t) f(y, t) d t d s+\sum_{l=1}^{N} \int_{a}^{b} \int_{c}^{d}(-1)^{l} r(s, \zeta) \frac{(\zeta-z)^{l}}{l !} f_{(0, l)}(s, z) d \zeta d s \\
& \left.+\sum_{j=1}^{M} \int_{a}^{b} \int_{c}^{d}(-1)^{j} r(v, t) \frac{(v-y)^{j}}{j !} f_{(j, 0)}(y, t) d t d v-f(y, z) Q(b, d)\right] \\
& =\int_{a}^{b} \int_{c}^{d}(-1)^{M+N} \bar{Q}^{(M, N)}(y, s, z, t) f_{(M+1, N+1)}(s, t) d t d s .
\end{aligned}
$$

We can easily get desired result by taking absolute value and by using inequality of Hölder for double integrals.

\section{Remark 3.4}

For $M=N=0$, we can obtain especial case of Theorem 3.3 as similar as Theorem 5 of (Pečarić and Vukelić, 2007)

\section{Double Weighted Integrals of Grüss type Inequalities for Differentiable Functions of Higher Order with two Variables}

Grüss (1935) proved remarkable integral inequality in 1935, which is as follows (see also [Mitrinović et al., 1991: 296]):

$$
\begin{aligned}
& \left|\frac{1}{b-a} \int_{a}^{b} f(y) g(y) d y-\left(\frac{1}{b-a} \int_{a}^{b} f(y) d y\right)\left(\frac{1}{b-a} \int_{a}^{b} g(y) d y\right)\right| \\
& \leq \frac{1}{4}(N-n)(M-m)
\end{aligned}
$$

provided that $g$ and $f$ are both integrable functions in the interval $[a, b]$ and satisfying the following:

$$
n \leq g(y) \leq N, m \leq f(y) \leq M, \forall y \in[a, b]
$$

where, $m, n, M, N$ real constants.

Pečarić and Vukelić, (2007), using identities (1.3) and (1.4) Peĉarić and Vukelić have given new Grüss type inequalites for double weighted integrals. Now by using differentiable functions of higher order for two variables, we get results for more generalization but for this purpose we use following notations for simplification instead of detailed presentations:

$$
\begin{aligned}
& B^{(j, l)}(y, z)=r(y, z)\left[f_{(j, l)}(y, z) g(y, z)\right. \\
& \left.+g_{(j, l)}(y, z) f(y, z)\right] Q_{\left(\begin{array}{c}
a \\
c
\end{array}\right) \Rightarrow\left(\begin{array}{c}
b \\
d
\end{array}\right)}^{(j)}(y, z), \\
& B(y, z)=r(y, z) \int_{a}^{b} \int_{c}^{d} r(s, t) \\
& {[f(s, t) g(y, z)+g(s, t) f(y, z)] d t d s,} \\
& \hat{B}^{(M, l)}(y, z)=r(y, z) \int_{a}^{b}\left[f_{(M+1, l)}(s, z) g(y, z)\right. \\
& \left.+g_{(M+1, l)}(s, z) f(y, z)\right] \hat{Q}^{(M, l)}(y, s, z) d s, \\
& \tilde{B}^{(j, N)}(y, z)=r(y, z) \int_{c}^{d}\left[g(y, z) f_{(j, N+1)}(y, t)\right. \\
& \left.+g_{(j, N+1)}(y, t) f(y, z)\right] \tilde{Q}^{(j, N)}(y, z, t) d t, \\
& \bar{B}^{(M, N)}(y, z)=r(y, z) \int_{a}^{b} \int_{c}^{d}\left[g(y, z) f_{(M+1, N+1)}(s, t)\right. \\
& \left.+g_{(M+1, N+1)}(s, t) f(y, z)\right] \times \bar{Q}^{(M, N)}(y, s, z, t) d t d s,
\end{aligned}
$$




$$
\begin{aligned}
& C^{(j, l)}(y, z)=\mid r(y, z) g(y, z)\left\|f_{(j, l)}(y, z)\right\|_{\infty} \\
& +\mid r(y, z) f(y, z)\left\|g_{(j, l)}(y, z)\right\| \|_{\infty}, \\
& \left.D^{(j, l)}(y, z)=\frac{(\max \{b-y, y-a\})^{j+1}}{(j+1) !} \frac{\max \{d-z, z-c\}) l+1}{(l+1) !} \int_{a}^{b} \int_{c}^{d} r r(v, \varsigma)\right) d \zeta d v,
\end{aligned}
$$

$D^{(0, l)}(z)=(b-a) \frac{(\max \{d-z, z-c\})^{l+1}}{(l+1) !} \int_{a}^{b} \int_{c}^{d} \mid r(v, \varsigma) d \varsigma d v$,

$D^{(j, 0)}(y)=(d-c)=\frac{(\max \{b-y, y-a\})^{j+1}}{(j+1) !} \int_{a}^{b} \int_{c}^{d} r(v, \varsigma) d \varsigma d v$,

$\tilde{D}^{(M, l)}(y, z)=\int_{a}^{b}\left|\hat{Q}^{(M, l)}(y, s, z)\right| d s$,

$\tilde{D}^{(j, N)}(y, z)=\int_{c}^{t}\left|\tilde{Q}^{(j, N)}(y, z, t)\right| d t$

$\bar{D}^{(M, N)}(y, z)=\int_{a}^{b} \int_{c}^{d}\left|\bar{Q}^{(M, N)}(y, s, z, t)\right| d t d s$,

$G_{f}(y, z)=R(f: y, z)+\int_{a}^{b} \int_{c}^{d} r(s, t) f(s, z) d t d s$

$+\int_{a}^{b} \int_{c}^{d} r(s, t) f(y, t) d t d s$

$+\sum_{l=1}^{N} \int_{a}^{b} \int_{c}^{d}(-1)^{l} r(s, \zeta) \frac{(\zeta-z)^{l}}{l !} f_{(0, l)}(s, z) d \zeta d s$

$+\sum_{j=1}^{M} \int_{a}^{b} \int_{c}^{d}(-1)^{j} r(v, t) \frac{(v-y)^{j}}{j !} f_{(j, 0)}(y, t) d t d v$,

$G_{g}(y, z)=R(g: y, z)+\int_{a}^{b} \int_{c}^{d} r(s, t) g(s, z) d t d s$

$+\int_{a}^{b} \int_{c}^{d} r(s, t) g(y, t) d t d s$

$+\sum_{l=1}^{N} \int_{a}^{b} \int_{c}^{d}(-1)^{l} r(s, \zeta) \frac{(\zeta-z)^{l}}{l !} g_{(0, l)}(s, z) d \zeta d s$

$+\sum_{j=1}^{M} \int_{a}^{b} \int_{c}^{d}(-1)^{j} r(v, t) \frac{(v-y)^{j}}{j !} g_{(j, 0)}(y, t) d t d v$,

where, $\hat{Q}^{(M, l)}(y, s, z), \tilde{Q}^{(j, N)}(y, z, t), \bar{Q}^{(M, N)}(y, s, z, t)$ are as in Theorem 2.2 whereas $Q$ and $R(f: y, z)$ are stated in (1.2) and (2.3) respectively.

Now, we are ready to get our important results of current section using notations defined above, which are as follows:

\section{Theorem 4.1}

Let $r, f, g:[a, b] \times[c, d] \rightarrow \mathbb{R}$ be three functions $\ni f, g$ $\in C^{(M+1, N+1)}([a, b] \times[c, d])$ and $r$ is an integrable. Then:

$$
\begin{aligned}
& \mid \frac{1}{Q(b, d)} \int_{a}^{b} \int_{c}^{l} r(y, z) f(y, z) g(y, z) d z d y \\
& -\left(\frac{1}{Q(b, d)} \int_{a}^{b} \int_{c}^{l} r(y, z) f(y, z) d z d y\right) \\
& \times\left(\frac{1}{Q(b, d)} \int_{a}^{b} \int_{c}^{d} r(y, z) g(y, z) d z d y\right) \mid \\
& \leq \frac{1}{2[Q(b, d)]^{2}} \int_{a}^{b} \int_{c}^{l}\left[\sum_{j=1}^{M} \sum_{l=1}^{N}\right. \\
& \times C^{(j, l)}(y, z) D^{(j, l)}(y, z) \\
& +\sum_{l=1}^{N} C^{(0, l)}(z) D^{(0, l)}(z)+\sum_{j=1}^{M} C^{(j, 0)}(y) D^{(j, 0)}(y) \\
& +C^{(M+1, l)}(y, z) \hat{D}^{(M, l)}(y, z)+C^{(j, N+1)}(y, z) \tilde{D}^{(j, N)}(y, z) \\
& \left.+C^{(M+1, N+1)}(y, z) \bar{D}^{(M, N)}(y, z)\right] d z d y .
\end{aligned}
$$

\section{Proof}

From (2.6) we have below identities:

$$
\begin{aligned}
& f(y, z) Q(b, d)=R(f: y, z)+\int_{a}^{b} \int_{c}^{d} r(s, t) f(s, t) d t d s \\
& +\sum_{l=0}^{N} \int_{a}^{b}(-1)^{M+1+l} \hat{Q}^{(M, l)}(y, s, z) f_{(M+1, l)}(s, z) d s \\
& +\sum_{j=0}^{M} \int_{c}^{l}(-1)^{j+N+1} \tilde{Q}^{(j, N)}(y, z, t) f_{(j, N+1)}(y, t) d t \\
& -\int_{a}^{b} \int_{c}^{d}(-1)^{M+N} \bar{Q}^{(M, N)}(y, s, z, t) f_{(M+1, N+1)}(s, t) d t d s
\end{aligned}
$$

$$
\begin{aligned}
& g(y, z) Q(b, d)=R(g: y, z)+\int_{a}^{b} \int_{c}^{t} r(s, t) g(s, t) d t d s \\
& +\sum_{l=0}^{N} \int_{a}^{b}(-1)^{M+1+l} \hat{Q}^{(M, l)}(y, s, z) g_{(M+1, l)}(s, z) d s \\
& +\sum_{j=0}^{M} \int_{c}^{t}(-1)^{j+N+1} \tilde{Q}^{(j, N)}(y, z, t) g_{(j, N+1)}(y, t) d t \\
& -\int_{a}^{b} \int_{c}^{d}(-1)^{N+M} \bar{Q}^{(M, N)}(y, s, z, t) g_{(M+1, N+1)}(s, t) d t d s
\end{aligned}
$$

$\forall(y, z) \in[a, b] \times[c, d]$. Multiply (4.15) by $r(y, z) g(y, z)$ and (4.16) by $r(y, z) f(y, z)$ and then summing these identities, we get:

$$
\begin{aligned}
& 2 Q(b, d) r(y, z) f(y, z) g(y, z) \\
& =-\sum_{j=1}^{M} \sum_{l=1}^{N}(-1)^{j+l} B^{(j, l)}(y, z)-\sum_{l=1}^{N}(-1)^{l} B^{(0, l)}(z) \\
& -\sum_{j=1}^{M}(-1)^{j} B^{(j, 0)}(y)+B(y, z)+(-1)^{M+1+l} \hat{B}^{(M, l)}(y, z) \\
& +(-1)^{j+N+1} \tilde{B}^{(j, N)}(y, z)-(-1)^{M+N} \bar{B}^{(M, N)}(y, z)
\end{aligned}
$$

Integrate the above equation over $[a, b] \times[c, d]$ and divided by $2 Q(b, d)$ on both sides and obtain: 


$$
\begin{aligned}
& \int_{a}^{b} \int_{c}^{d} f(y, z) g(y, z) r(y, z) d z d y \\
& =\frac{1}{2 Q(b, d)} \int_{a}^{b} \int_{c}^{d}\left[-\sum_{j=1}^{M} \sum_{l=1}^{N}(-1)^{j+l} B^{(j, l)}(y, z)\right. \\
& -\sum_{l=1}^{N}(-1)^{l} B^{(0, l)}(z)-\sum_{j=1}^{M}(-1)^{j} B^{(j, 0)}(y)+B(y, z) \\
& +(-1)^{M+1+l} \hat{B}^{(M, l)}(y, z)+(-1)^{j+N+1} \tilde{B}^{(j, N)}(y, z) \\
& \left.-(-1)^{N+M} \bar{B}^{(M, N)}(y, z)\right] d z d y
\end{aligned}
$$

It may be written as:

$$
\begin{aligned}
& \frac{1}{Q(b, d)} \int_{a}^{b} \int_{c}^{l} r(y, z) f(y, z) g(y, z) d z d y \\
& -\left(\frac{1}{Q(b, d)} \int_{a}^{b} \int_{c}^{l} f(y, z) r(y, z) d z d y\right) \\
& \times\left(\frac{1}{Q(b, d)} \int_{a}^{b} \int_{c}^{l} r(y, z) g(y, z) d z d y\right) \\
& =\frac{1}{2[Q(b, d)]^{2}} \int_{a}^{b} \int_{d}^{l}\left[-\sum_{j=1}^{M} \sum_{l=1}^{N}(-1)^{j+l} B^{(j, l)}(y, z)\right. \\
& -\sum_{l=1}^{N}(-1)^{l} B^{(0, l)}(z) \\
& -\sum_{j=1}^{M}(-1)^{j} B^{(j, 0)}(y)+(-1)^{M+1+l} \hat{B}^{(M, l)}(y, z) \\
& \left.+(-1)^{j+N+1} \tilde{B}^{(j, N)}(y, z)-(-1)^{M+N} \bar{B}^{(M, N)}(y, z)\right] d z d y .
\end{aligned}
$$

Using (4.1),..,(4.12) we get below inequalities $\forall(y, z)$ $\in[a, b] \times[c, d]$

$$
\begin{aligned}
& \left|(-1)^{j+l} B^{(j, l)}(y, z)\right| \leq C^{(j, l)}(y, z) D^{(j, l)}(y, z), \\
& \left|(-1)^{l} B^{(0, l)}(z)\right| \leq C^{(0, l)}(z) D^{(0, l)}(z), \\
& \left|(-1)^{j} B^{(j, 0)}(y)\right| \leq C^{(j, 0)}(y) D^{(j, 0)}(y), \\
& \left|(-1)^{M+1+l} \hat{B}^{(M, l)}(y, z)\right| \leq C^{(M+1, l)}(y, z) \hat{D}^{(M, l)}(y, z), \\
& \left|(-1)^{j+N+1} \tilde{B}^{(j, N)}(y, z)\right| \leq C^{(j, N+1)}(y, z) \tilde{D}^{(j, N)}(y, z), \\
& \left|(-1)^{M+N} \bar{B}^{(M, N)}(y, z)\right| \leq C^{(M+1, N+1)}(y, z) \bar{D}^{(M, N)}(y, z) .
\end{aligned}
$$

We can easily get desired result by taking absolute value in (4.18) on both sides and by using above these inequalites in it.

\section{Theorem 4.2}

Let $r, f, g:[a, b] \times[c, d] \rightarrow \mathbb{R}$ be three functions $\ni$ $f, g \in C^{(M+1, N+1)}$ in the same interval and $r$ is an integrable. Then:

$$
\begin{aligned}
& \mid \frac{1}{Q(b, d)} \int_{a}^{b} \int_{c}^{d} r(y, z) f(y, z) g(y, z) d z d y \\
& +\left(\frac{1}{Q(b, d)} \int_{a}^{b} \int_{c}^{d} r(y, z) f(y, z) d z d y\right)\left(\frac{1}{Q(b, d)} \int_{d}^{b} \int_{d}^{d} r(y, z) g(y, z) d z d y\right) \\
& -\frac{1}{2[Q(b, d)]^{2}} \int_{a}^{b} \int_{r}^{l} r(y, z)\left[g(y, z) G_{f}(y, z)+f(y, z) G_{g}(y, z)\right] d z d y \mid \\
& \leq \frac{1}{2[Q(b, d)]^{2}} \int_{a}^{b} \int_{d}^{l} C^{(M+1, N+1)}(y, z) \bar{D}^{(M, N)}(y, z) d z d y
\end{aligned}
$$

Proof

From (2.7) we have below identities:

$$
\begin{aligned}
& f(y, z) Q(b, d)=G_{f}(y, z)-\int_{a}^{b} \int_{c}^{d} r(s, t) f(s, t) d t d s \\
& +\int_{a}^{b} \int_{c}^{d}(-1)^{N+M} \bar{Q}^{(M, N)}(y, s, z, t) f_{(M+1, N+1)}(s, t) d t d s, \\
& g(y, z) Q(b, d)=G_{g}(y, z)-\int_{a}^{b} \int_{c}^{d} r(s, t) g(s, t) d t d s \\
& +\int_{a}^{b} \int_{c}^{d}(-1)^{N+M} \bar{Q}^{(M, N)}(y, s, z, t) g_{(M+1, N+1)}(s, t) d t d s,
\end{aligned}
$$

for $(y, z) \in[a, b] \times[c, d]$. Multiply (4.19) by $r(y, z) g(y, z)$ and (4.20) by $r(y, z) f(y, z)$ and then summing these identities, we get:

$$
\begin{aligned}
& 2 Q(b, d) r(y, z) f(y, z) g(y, z) \\
& =r(y, z) g(y, z) G_{f}(y, z) \\
& +r(y, z) f(y, z) G_{g}(y, z) \\
& -B(y, z)+(-1)^{M+N} B^{(M+N)}(y, z)
\end{aligned}
$$

Integrate the above equation over $[a, b] \times[c, d]$ and divided by $2 Q(b, d)$ on both sides and obtain:

$$
\begin{aligned}
& \int_{a}^{b} \int_{c}^{d} r(y, z) f(y, z) g(y, z) d z d y \\
& =\frac{1}{2 Q(b, d)} \int_{a}^{b} \int_{c}^{d} r(y, z)\left[g(y, z) G_{f}(y, z)+f(y, z) G_{g}(y, z)\right] d z d y \\
& -\frac{1}{Q(b, d)}\left(\int_{a}^{b} \int_{c}^{d} f(y, z) r(y, z) d z d y\right)\left(\int_{a}^{b} \int_{c}^{d} g(y, z) r(y, z) d z d y\right) \\
& +\frac{1}{2 Q(b, d)} \int_{a}^{b} \int_{c}^{d}(-1)^{M+N} \bar{B}^{(M, N)}(y, z) d z d y .
\end{aligned}
$$

since we have:

$\left|(-1)^{M+N} \bar{B}^{(M, N)}(y, z)\right| \leq C^{(M+1, N+1)}(y, z) \bar{D}^{(M, N)}(y, z)$.

From (4.22) and (4.23) we obtain our required inequality. 


\section{Remark 4.3}

For $N=M=0$, we can obtain especial cases of Theorems 4.1 and 4.2 as similar as Theorems 6 and 7 of (Pečarić and Vukelić, 2007) respectively, we may also obtain as similar results as in (Guezane-Lakoud and Aissaoui, 2011).

\section{Conclusion}

In the last section of this article, we would present our conclusion of this article is that we gave the more generalized identities of Montgomery for differentiable function of higher order for two independent variables as compare to Pečarić and Vukelić (2007) results which were given in (Pečarić and Vukelić, 2007) and we gave especial cases of identities of Montgomery by putting $r(s, t)=q(s) p(t)$. We also obtained more generalized Ostrowski and Grüss type inequalities for differentiable functions of higher order for two independent variables by the help of generalized identities of Montgomery as compare to the results of (Pečarić and Vukelić, 2007).

\section{Author's Contributions}

All authors equally contributed in this work.

\section{Ethics}

This article is original and contains unpublished material. The corresponding author confirms that all of the other authors have read and approved the manuscript and no ethical issues involved.

\section{References}

Adnan, M., R.K. Asif and F. Mehmood, 2017. Positivity of sums and integrals for higher order $\Delta$-convex and completely monotonic functions. Classical Analysis ODEs (math.CA), arXiv:1710.07182v1

Alomari, M. and M. Darus, 2010a. Some ostrowski type inequalities for convex functions with applications. RGMIA13.

Alomari, M. and M. Darus, 2010b. Some ostrowski type inequalities for quasi-convex functions with applications to special means. RGMIA13.

Barnett, N.S. and S.S. Dragomir, 2001. An ostrowski type inequality for double integrals and applications for cubature formulae, Soochow J. Math., 27: 1-10.

Buse, C., P. Cerone, S.S. Dragomir, J. Roumeliotis, 2006. A refinement of Grüss type inequality for the bochner integral of vector-valued functions in hilbert spaces and applications. J. Korean Math. Soc., 43: 911-929.
Cerone, P. and S.S. Dragomir, 2007. A Renment of the Grüss inequality and applications. Tamkang J. Math. 38: 37-49.

Dragomir, S.S. and T.M. Rassias, 2002. Ostrowski Type Inequalities and Applications in Numerical Integration. 1st Edn., Netherlands, ISBN-10: 978-94-017-2519-4, pp: 482.

Dragomir, S.S., 2012. New Grüss' type inequalities for functions of bounded variation and applications. Appl. Math. Lett. 25: 1475-1479.

Dragomir, S.S., N.S. Barnett and P. Cerone, 2003. An ostrowski type inequality for double integrals in terms of Lp-norms and applications in numerical integration. Rev. Anal. Numer. Theor. Approx., 32 : 161-169.

Dragomir, S.S., P. Cerone, N.S. Barnett and J. Roumeliotis, 2000. An inequality of the ostrowski type for double integrals and applications for cubature formulae, Tamsui. Oxf. J. Math. Sci. 16: 1-16.

Dragomir, S.S., Y.J. Cho, S.S. Kim and Y. Kim, 2008. On Bessel's and Grss inequalities for orthonormal families in 2-inner product spaces and applications. Kyungpook Math. J., 48: 207-222.

Grüss, G., 1935. Über das Maximum des Absoluten Betrages

von

$[1 /(b-a)] \int_{a}^{b} f(x) g(x) d x-\left[1 /(b-a)^{2}\right] \int_{a}^{b} f(x) d x \int_{a}^{b} g(x) d x$

Math. Z. 39: 215-226.

Guezane-Lakoud, A. and F. Aissaoui, 2011. New Čebyšev's type inequalitities for double integrals. J. Math. Ineq., 5: 453-462.

Liu, W., 2012. New bounds for the companions of ostrowski's inequality and applications.

Mitrinović, D.S., J.E. Pečarić and A.M. Fink, 1991. Inequalities Involving Functions and Their Integrals and Derivatives.1st Edn., United States, ISBN-10: 9401135630, pp: 606.

Ostrowski, A., 1938. Über die Absolutabweichung einer differentiebaren Funktion von ihren Integralmittelwert, Comment. Math. Helv. 10: 226-227.

Pečarić, J., 1980. On the Čebyšev's Inequality, Bull. Inst. Politeh. Timisoara.

Pečarić, J.E. and A. Vukelić, 2007. Montgomery's Identities for functions of two variables, J. Math. Anal. Appl. $332: 617-630$.

Pečarić, J.E., 1984. On the Ostrowski's Generalization of Čebyšev's Inequality, J. Math. Anal. Appl. 102: 479-487.

Pečarić, J.E., 1987. Some Further Remarks on the Ostrowski's Generalization of Čebyšev's Inequality. J. Math. Anal. Appl. 123: 18-33. 